likely developed, since the patient had been exposed to excess epinephrine for a long time. It has been shown that cyclic AMP increase due to epinephrine administration is impaired in asthmatics. Asthmatic patients have decreased betaadrenergic receptor "availability" and thus betaadrenergic dysfunction. ${ }^{6.7}$ Furthermore, the withdrawal of the effect of prazosin which was given preoperatively, may trigger the recurrence of asthma, because alpha-blockade has been shown to increase cyclic AMP in asthmatic patients ${ }^{8}$ and to inhibit the bronchoconstriction by alpha-agonists. ${ }^{9}$

In summary, since adrenal secretion of epinephrine plays an important role in the relaxation of bronchial smooth muscles, we should keep in mind that episodes of bronchial constriction can occur after adrenalectomy in asymptomatic asthmatic patients.

Toshiaki Nishikawa, MD

Shuji Dohi, MD

Yukinobu Anzai, MD

Department of Anesthesiology

Sapporo Medical College

South-1, West-16, Chuoku, Sapporo 060

Japan

and

Department of Anesthesiology

Institute of Clinical Medicine

University of Tsukuba, Japan

REFERENCES

1 Leff $A R$. Pathogenesis of asthma. Neurophysiology and pharmacology of bronchospasm. Chest 1982; 81: 224-9.

2 Aviado DM. Regulation of bronchomotor tone during anesthesia. Anesthesiology 1975; 42: 68-80.

3 Hirshman CA. Airway reactivity in humans. Anesthetic implications. Anesthesiology 1983; 58 : 170-7.

4 Harvey JN, Dean HG, Lee MR. Recurrence of asthma following removal of a noradrenalinesecreting phaeachromocytoma. Postgrad Med J 1984; 60: 364-5.

5 Coret IA, VanDyke $H B$. The altered blood-pressure response after adrenolytic drugs and large doses of sympathomimetic amines. J Pharmacol Exp Ther 1949; 95: 415-20.

6 Makino S, Ikemori R, Fukuda T, Motojima S. Relationships between responsiveness of the bronchi to acetylcholine and cyclic AMP response of lym- phocytes to beta $a_{1}$ - and beta $a_{2}$-adrenergic receptor stimulation in patients with asthma. Allergy 1983; 38: 37-42.

7 Motojima S, Fukuda T, Makino S. Measurement of beta-adrenergic receptors on lymphocytes in normal subjects and asthmatics in relation to betaadrenergic hyperglycaemic response and bronchial responsiveness. Allergy 1983; 38: 331-7.

8 Kitamura S, Ishihara Y, Yotsumoto $H$, Kosaka $K$. The potentiating effects of an alphaadrenergic blocking agent (E-643) on the action of bronchodilators. Drug Res 1980; 30: 37-40.

9 Leff AR, Munoz N. Evidence for two subtypes of alpha adrenergic receptors in canine airway smooth muscle. J Pharmacol Exp Ther 1981; 217: $530-5$.

\section{Abnormal ECG pattern during battery operation of Mennen model 741 ECG monitor}

To the Editor:

We wish to report a problem which occurred during our use of a recently acquired Mennen model 741 ECG Monitor (Mennen Medical Inc., Clarence, New York). During battery operation the monitor produced an abnormal wave form which incorrectly suggested a sudden change in the patient's status.

The monitor was being used during a general anaesthetic for hysterectomy in an ASA physical status 1 patient. The ECG indicated normal sinus rhythm for approximately one hour until it suddenly began to indicate an abnormal ECG pattern with widened QRS complexes and deep inverted ST segments, suggestive of ischaemia (Figure). The patient's clinical status appeared stable and satisfactory. Heart rate was $80 \mathrm{bpm}$ and blood pressure $120 / 70 \mathrm{mmHg}$, both unchanged from previous values during the normal ECG pattern. A careful search for other factors which might lead to the abnormal ECG pattem proved negative.

After about 20 minutes it was realized that the electrical socket into which the monitor had been plugged did not have a power supply, and that therefore the monitor had been operating, from the outset, from its battery supply. The monitor was then plugged into an active electrical socket, which 


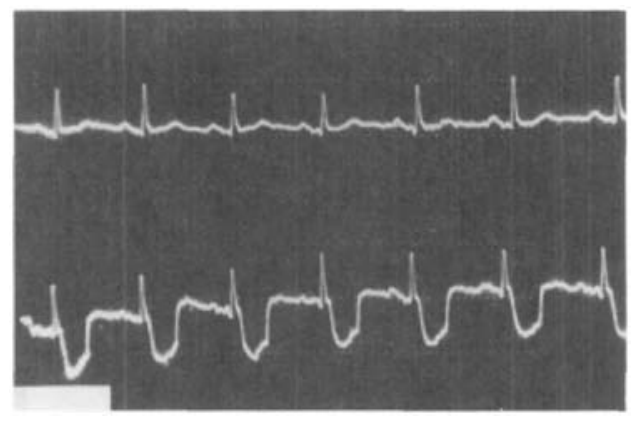

FIGURE Normal ECG pattem, during operation from line power (top). Abnormal pattem, during operation from battery (bottom).

immediately restored the ECG pattern to normal. The abnormal ECG pattern was reproduced when the monitor was plugged back into the original socket, thereby restoring operation from the battery.

Our Model 741 monitor includes a red indicator which is supposed to light when the battery voltage drops below $11.2 \mathrm{~V}$ (the normal battery output is $12.0 \mathrm{~V}$ ). This indicator did not light during the event described above.

Since encountering this problem we have become aware that a similar problem was reported to occur with a Mennen model 742 ECG monitor.' This monitor was noted to display inaccurate heart rate and spurious wave forms resembling ventricular tachycardia and other cardiac arrhythmias when operating under low battery conditions. This occurred when the monitor's battery voltage dropped below $9.5 \mathrm{~V}$. The red low-battery indicator did activate, in contrast to our experience.

The Health Devices report indicates that there are five Mennen series 700 portable ECG monitors with optional battery power (Models 740, 741, 742, 743 and 744). The report recommends that hospitals with Mennen 700 series portable monitors should contact the Manager of Technical Services, Mennen Medical at 716-759-6921 to obtain warning labels free of charge for the low battery indicator. These labels state: "Red LED indicates low battery. Return to AC power." The Health Devices report further suggets that an additional label should be attached to series 700 monitors: "Warning: Low battery operation may result in erroneous displays."

It is clearly essential that anaesthetists who use a monitor with a built-in tendency to produce an abnormal wave form in some circumstances must be completely aware of this possibility, in order to prevent unnecessary and potentially dangerous treatment being initiated in response to an abnormal ECG pattern. Our experience also serves as a reminder of another important basic premise: treat the patient, not the monitor.

N.V. Palkar, MB, BS, MD (Anaesth)

H. Moseley, FFARCs (London)

Department of Anaesthesia

Queen Elizabeth Hospital

Martindales Road

St. Michael, Barbados (W.I.)

REFERENCE

1 Mennen Series 70 Portable Monitor. Health Devices $1985 ; 14 ; 134-5$

\section{Contralateral Horner's Syndrome following stellate ganglion block}

To the Editor:

We would like to report an unusual complication of a stellate ganglion block performed on a 63-yearold patient for the treatment of a reflex sympathetic dystrophy.

A contralateral Horner's Syndrome occurred after the seventh in a series of 15 left stellate blocks. The technique used was identical to the previous six blocks. The stellate ganglion blocks were performed with the patient supine, using an anterior approach with the anterior tubercle of $\mathrm{C} 6$ as a bony landmark.' A $3.75 \mathrm{~cm} 22$-gauge needle and 7-8 cc of 0.25 per cent plain bupivacaine were used to perform the blocks. With this technique, the patient rapidly developed a left Horner's syndrome following each block.

Following the seventh block, the patient developed a right-sided ptosis and meiosis, which subsequently became a complete right Homer's syndrome. The patient had no evidence of subarachnoid block, hoarsenes or neurologic deficits in either arm. Subsequent blocks were followed by ipsilateral Homer's syndromes.

Warrick reported one case of a contralateral Horner's syndrome from a stellate ganglion block, 